\title{
Graue Literatur
}

Das Wiki zur Lesbengeschichte der Schweiz: Conti-Club, https://l-wiki.ch/Conti-Club vom 16.12.2020.

—: https://l-wiki.ch/L-World_-_Das_Wiki_zur_Lesbengeschichte_der_Schweiz vom 16.12.2020.

Die Siegessäule: CSD in Berlin. Konflikt und Konsens: Die Siegessäule-Podiumsdiskussion 2014, https://www.siegessaeule.de/magazin/1011-konflikt-und-konsens-die-si egess\%C3\%A4ule-podiumsdiskussion/ vom 20.10.2020.

Duden: Sensibilität, https://www.duden.de/rechtschreibung/Sensibilitaet vom 24.11. 2020.

Gladt e.V: Stellungnahme GLADT e.V. zum Kiss In von Maneo am 17. Mai Internationaler Tag gegen Homophobie und Trans"phobie 2015, http://dkp-queer.de/stellun gnahme-gladt-e-v-zum-kiss-in-von-maneo-am-17-mai-internationaler-tag-gegen -homophobie-und-transphobie/9158 vom 24.11.2020.

HFG: Homosexuelle Frauengruppe Zürich. Lesbenfront 1975, https://www.e-periodica. ch/digbib/view?pid=les-001\%3A1975\%3A0\#5 vom 16.12.2020.

—: Lesbenkampf ist Frauenkampf 1976.

Hosi Wien: Lambda-Nachrichten, https://www.lambdanachrichten.at/ vom 15.09. 2020.

Jugendnetzwerk Lambda: Endlich Volljährig. Lambda wird 18, Out! 2008.

—: »Geoutet Zuhause wohnen«, in: Wohnen, Out! 2010.

-: Wohnen, Out! 2010.

—: „Denkwürdig«, in: Internationalität, Out! 2011.

—: CSD - Politik oder Konsum«, in: Konsum, Out! 2012.

—: Träume, Out! 2013.

Jugendnetzwerk Lambda:BB: Beratung, https://www.lambda-bb.de/beratung vom 20. 11.2020 .

—: Coming-out, https://www.lambda-bb.de/beratung/coming-out vom 24.11.2020.

—: Filmprojekte, https://www.lambda-bb.de/presse-material/filmprojekte vom 20.11. 2020.

—: In\&Out-Beratung, https://comingout.de/ vom 20.08.2020.

—: Jugendgruppen, https://www.lambda-bb.de/jugendgruppen vom 20.11.2020. 
—: Lambda ${ }^{2}$ Projekt, https://www.lambda-bb.de/projekte/lambda\%c2\%b2-projekt vom 20.08.2020.

—: Podcasts, https://www.lambda-bb.de/presse-material/podcasts vom 20.11.2020.

—: Pressemitteilungen, https://www.lambda-bb.de/presse-material/pressematerial vom 20.08.2020.

—: Pride Café, https://www.lambda-bb.de/pridecafe vom 20.11.2020.

—: Printmaterialien, https://www.lambda-bb.de/presse-material/printmaterialien vom 20.08.2020.

—: queer@school, https://queer-at-school.de/ vom 20.08.2020.

—: Queeres Jugendzentrum, https://www.lambda-bb.de/projekte/queeres-jugendzent rum vom 20.11.2020.

—: Studien, https://www.lambda-bb.de/studien-2 vom 20.08.2020.

-: Unsere Geschichte, https://www.lambda-bb.de/uber-uns/unsere-geschichte vom 20.11.2020.

-: ... Eröffnung erstes queeres Jugendhaus in Berlin! 2014, https://www.lambda-bb.de /wp-content/uploads/2014/04/Pressemitteilung-02-2014.pdf vom 20.08.2020.

-: Queeres Jugendhaus in Pankow! 2014, https://www.lambda-bb.de/wp-content/uplo ads/2013/09/Pressemitteilung-04-2013.pdf vom 20.11.2020.

—: Pressemitteilung Nr. 03-2015 ... zu Roland Emmerichs Film »Stonewall« 2015, http s://www.lambda-bb.de/wp-content/uploads/2012/12/StellungnahmeStonewall.pdf vom 23.11.2020.

-: Stellungnahme des Jugendnetzwerks Lambda Berlin-Brandenburg auf dem Berliner Christopher Street Day am 27. Juni 2015, https://www.lambda-bb.de/wp-content/u ploads/2012/12/Stellungnahme-Lambda-zu-CSD_030820105-.pdf vom 20.11.2020.

-: Stellungnahme zur Nicht-Teilnahme der Lesbenberatung/LesMigraS am LesbischSchwulen Stadtfest 2015, https:/www.lambda-bb.de/wp-content/uploads/2015/0 6/Stellungnahme_Nicht-Teilnahme-Lesbenberatung_FINAL_18062015.pdf vom 23. 11.2020.

-: Berliner Senat beschließt Konzept für queeres Jugendzentrum! 2018, https://www. lambda-bb.de/wp-content/uploads/2018/02/2018-02-16-PM-Konzept-QJZ.pdf vom 13.03.2020.

-: Zur Vergabe des queeren Jugendzentrums an Lambda BB 2020, https://www.lamb da-bb.de/wp-content/uploads/2018/06/2018-06-08-Pressemitteilung-Vergabe-que eres-Jugendzentrum.pdf vom 13.03.2020.

Landesverband AndersARTiG e.V.: Die LesBI*Schwule T*our. Die Kampagne, http://br andenburg-bleibt-bunt.de/die-kampagne.html vom 13.03.2020.

LesBi* SchwuleT*our: Tour-Fibel, http://brandenburg-bleibt-bunt.de/die-tour-fibel/die -regenbogenfahne.html vom 20.08.2020.

LesMigraS: Antirassismus und LSBTIQ https://lesmigras.de/antirassismus-und-lsbtiq .html vom 18.03.2020.

—: Archiv, https://lesmigras.de/Archiv.html vom 15.09.2020.

—: Beratung, https://lesmigras.de/beratung.html vom 13.03.2020.

-: Chronologie der Antidiskriminierungs- und Antigewaltarbeit der Lesbenberatung, https://lesmigras.de/25-jahre-antidiskriminierungsantigewaltarbeit.html vom 15.09.2020. 
—: Diskriminierungssensibilität, https://lesmigras.de/diskriminierungssensibilitaet.h tml vom 13.03.2020.

—: Förderung, https://lesmigras.de/foerderung.html vom 15.09.2020.

—: Gewaltrad, https://lesmigras.de/gewaltrad.html vom 13.03.2020.

—: Gewaltverständnis, https://lesmigras.de/tl_files/lesmigras/Tapesh/Texte/2015_Gew altverstaendnis.pdf vom 26.08.2020.

—: Studie, LesMigraS, https://lesmigras.de/studie_mehrfachdiskriminierung.html vom 13.03.2020.

—: Tapesh, https://lesmigras.de/tapesh.html vom 20.08.2020.

—: Tapesh_Angebote, https://lesmigras.de/tapesh_angebote.html vom 13.03.2020.

—: Team, https://lesmigras.de/team.html vom 20.08.2020.

—: Workshops und Events, https://lesmigras.de/workshops_und_events.html vom 13.03.2020.

-: Russische Lesben in Europa, Berlin 2004.

-: Stimmen arabischer Lesben - Arabische Lesben in Deutschland und Europa, Berlin 2004.

—: Zeichen setzen! Türkeistämmige Lesben in Deutschland und Europa, Berlin 2004.

-: Leben iranischer Lesben - Iranische Lesben in Deutschland und Europa, Berlin 2006.

—: Klares Signal gegen Rassismus. Judith Butler lehnt Zivilcourage-Preis des BerlinerCSD e.V. ab. 2010, https://lesmigras.de/tl_files/lesmigras/pressemitteilungen/Stell ungnahme_LB_Butler.pdf vom 26.09.2020.

-: Unterstützung geben. Handlungsstrategien im Umgang mit Gewalt und Diskriminierung. 2011, https://lesmigras.de/tl_files/lesmigras/Tapesh/LM_Broschuere_Tap esh_UnterstuetzungGeben.pdf vom 13.03.2020.

-: Verbindungen sprechen. Empowerment in Bezug auf Rassismus und Transphobie in LSBTI-Kontexten 2011, https://lesmigras.de/tl_files/lesmigras/Tapesh/LM_Brosc huere_Tapesh_einzelseiten_korrigiert.pdf vom 20.08.2020.

- : >... nicht so greifbar und doch real. Eine quantitative und qualitative Studie $\mathrm{zu}$ Gewalt- und (Mehrfach-)Diskriminierungserfahrungen von lesbischen, bisexuellen Frauen und Trans* in Deutschland, Berlin 2012, https://lesmigras.de/tl_files/lesbe nberatung-berlin/Gewalt(Dokus,Aufsätze...)/DokumentationStudieweb_sicher.pdf vom 20.08.2020.

-: Handlungsmöglichkeiten bei Gewalt in Beziehungen 2012.

—: Informationen zum Beratungs-Angebot in leichter Sprache 2012.

—: Redebeitrag LesMigraS auf TCSD 2012, https://lesmigras.de/tl_files/lesmigras/pres semitteilungen/Redebeitrag_LesMigraS_TCSD_2012.pdf vom 13.03.2020.

—: Was ist Mehrfachzugehörigkeit und Mehrfachdiskriminierung? 2012, https://lesmi gras.de/tl_files/lesmigras/Tapesh/Texte/2015_MFD.pdf vom 20.11.2020.

—: Internationaler Coming-Out-Tag am 11.10.2013. Pressemitteilung der Lesbenberatung e.V. Berlin 2013, https://lesmigras.de/tl_files/lesmigras/pressemitteilungen/P M_2013_Coming_Out.pdf vom 13.03.2020.

—: Rede/Audiobeitrag von LesMigraS auf dem TCSD 2013, https:/lesmigras.de/t 1_files/lesmigras/pressemitteilungen/Redebeitrag_LesMigraS_TCSD_2013.pdf vom 20.11.2020. 
—:Wir erklären Mehrfach-Zugehörigkeit und Mehrfach-Benachteiligung. Text in leichter Sprache 2013.

—: Politik ist mehr als ein Name. Statement zur Umbenennung des Berliner CSD in Stonewall 2014, https://lesmigras.de/tl_files/lesmigras/pressemitteilungen/Statem ent_CSD_Stonewall_2014.pdf vom 13.03.2020.

-: Pressemitteilung anlässlich des Christopher Street Days. Mehrdimensionales Empowerment statt eindimensionaler Feiertag 2014, https://lesmigras.de/tl_files/lesm igras/pressemitteilungen/PM_2014_CSD.pdf vom 26.09.2020.

-: Pressemitteilung der Lesbenberatung e.V. Berlin zum Internationalen Tag gegen Homophobie und Trans*diskriminierung 17. Mai 2015, https://lesmigras.de/tl_files /lesmigras/pressemitteilungen/PM_IDAHOT_2015_LB.pdf vom 13.08.2020.

—: Redebeitrag von LesMigraS auf dem KCSD 2015, https://lesmigras.de/tl_files/lesmi gras/pressemitteilungen/Redebeitrag_LesMigraS_KCSD_2015.pdf vom 20.11.2020.

—: Stellungnahme zum Lesbisch-Schwulen Stadtfest 2015, https://lesmigras.de/tl_file s/lesbenberatung-berlin/Newsletter_/Stellungnahme_2015_Stadtfest_Lesbenbera tung.pdf vom 13.03.2020.

-: 10 PORTRÄTS - Lesbische, Schwule, Bisexuelle und Trans* Geflüchtete in Deutschland. 2017.

—: Grenzen setzen - Ein Arbeitsbuch 2017 vom https://lesmigras.de/tl_files/lesmigras /Texte/LesMigraS_Grenzen_Setzen_online.pdf.

Milchjugend: App, https://app.milchjugend.ch/ vom 15.09.2020.

—: Lila-Festival, https://lila-festival.ch/infos vom 13.03.2020.

—: Milchbar, https://milchjugend.ch/milchbar vom 13.03.2020.

—: Milchkultur, https://milchjugend.ch/milchkultur vom 13.03.2020.

—: Milchreise, https://milchjugend.ch/milchreise vom 13.08.2020.

-: Spenden, https://milchjugend.ch/spenden vom 13.08.2020.

—: Über die Milchjugend, https://milchjugend.ch/ueber-die-milchjugend vom 12.08. 2020.

—: Wir machen Druck, Milchbüechli 2012.

—:»DAS IST ER!«, in: drei und mehr, Milchbüechli 2013.

—: »Denk mal nach: Bringt's die Pride?!«, in: Triff uns Live, Milchbüechli 2013.

—: drei und mehr, Milchbüechli 2013.

-: Triff uns Live, Milchbüechli 2013.

—: »Weiss ist eine Hautfarbe«, in: An der Oberfläche, Milchbüechli 2013.

-: Hauptgang, Milchbüechli 2014.

—: Schlüsselerlebnis, Milchbüechli 2015.

—: Ausgabe 19, Milchbüechli 2017.

-: Ausgabe 20, Milchbüechli 2017.

-: Ausgabe 22, Milchbüechli 2017.

-: Ausgabe 24, Milchbüechli 2018.

—: Rechnung Milchjugend 20182019.

Netzwerk diskriminierungsfreie Szene für alle: Das Netzwerk, https://diskriminierung sfreieszenenfueralle.wordpress.com/netzwerke/ vom 24.11.2020.

Ohms, Constance/Müller, Karin: Gut aufgehoben? Zur psychosozialen Versorgung lesbischer Frauen mit Gewalt und/oder Diskriminierungserfahrungen im europäi- 
schen Vergleich 2001, https://lesmigras.de/tl_files/lesbenberatung-berlin/Gewalt\% 20(Dokus,Aufsaetze...)/gut_aufgehoben.pdf vom 20.08.2020.

Ostertag, Ernst/Rapp, Röbi: Schwulengeschichte, https://schwulengeschichte.ch/home/ vom 15.09.2020.

Perverse Partei Österreich: Die Perversen, https://www.facebook.com/DiePerversen/ vom 15.09.2020.

Romanescos: Über uns, https://www.romanescos.ch/Ueber-uns/ vom 28.11.2020.

Saadat-Lendle, Saideh: »Kategorien? Nein Danke! Saideh Saadat-Lendle über die Arbeit von LesMigraS«, in: »Nicht jammern, sondern klagen!«. Diskriminierungsschutz und Recht für Lesben, Schwule und Trans*, S. 19-24.

—: Pressekonferenz zur Vorstellung erster Ergebnisse der bisher umfangreichsten Studie zu Gewalt- und Mehrfachdiskriminierungserfahrungen von lesbischen/bisexuellen Frauen und Trans*. LesMigraS 2012, https://lesmigras.de/tl_files/lesmigras/p ressemitteilungen/Redebeitrag\%20Saideh\%20Saadat-Lendle.pdf vom 20.08.2020.

TransInterQueer e.V.: Begleitforschung bei Trans* Visible, https://www.transinterquee r.org/wp-content/uploads/Begleitforschung.pdf vom 20.08.2020.

—: Das »I« in TrIQ https:/www.transinterqueer.org/ueber-triq/das-i-in-triq/ vom 27.08.2020.

—: Fortbildungen \& Workshops, https://www.transinterqueer.org/thema/fortbildung/ vom 27.08.2020.

—: Gruppen, https://www.transinterqueer.org/gruppen/ vom 27.08.2020.

—: Inter*Projekt, https://interprojekt.wordpress.com/ vom 15.09.2020.

—: Kunst \& Kultur, https://www.transinterqueer.org/thema/kunst-kultur/ vom 27.08. 2020.

—: Öffentlichkeitsarbeit und Medien, https://www.transinterqueer.org/thema/offentli chkeitsarbeit-medien/ vom 27.08.2020.

—: Personenstand, https://www.transinterqueer.org/pstg/ vom 27.08.2020.

—: Persönliche Trans* - und Inter*-Beratung, https://www.transinterqueer.org/beratu ng/persoenliche-trans-inter-beratung/ vom 15.09.2020.

—: Politik \& Lobbyarbeit, https://www.transinterqueer.org/thema/politik-lobbyarbeit/ vom 15.09.2020.

—: Publikationen, https://www.transinterqueer.org/unsere-publikationen/ vom 27.08. 2020.

—: Refugees@TrIQ, https://www.transinterqueer.org/refugees/vom 27.08.2020.

—: Sozialberatung, https:/www.transinterqueer.org/beratung/sozialberatung/ vom 15.09.2020.

—: Thema aktuell, https://www.transinterqueer.org/thema/aktuell/ vom 15.09.2020.

—: Trans* Visible, https://www.transinterqueer.org/projekte/trans-visible/ vom 15.09. 2020.

—: Trans*Inter*Queer* ABC, https://www.transinterqueer.org/download/Publikation en/TrIQ-ABC_web(2).pdf vom 20.11.2020.

-: Transgenderradio, https://www.transinterqueer.org/projekte/transgenderradio/ vom 27.08.2020.

—: Über den Verein TransInterQueer, https://www.transinterqueer.org/ueber-triq/ver ein/ vom 27.08.2020. 
—: Unterstützer_innen, https://www.transinterqueer.org/ueber-triq/unterstuetzer_in nen/ vom 27.08.2020.

—:Warum dieses Projekt gebraucht wird, https://www.transinterqueer.org/ueber-triq /warum-dieses-projekt-gebraucht-wird/ vom 27.08.2020.

-: Wie funktioniert TrIQ?, https://www.transinterqueer.org/ueber-triq/wie-funktioni ert-triq/ vom 27.08.2020.

-: ... And Others! Argumentation Training for Transgender Inclusion in Europe. A >Good Practice< Toolkit for Trans* Activists and Allies Working for Trans* Equality, Rights and Inclusion. 2011, https:/www.transinterqueer.org/download/Publik ationen/Transgender_Inclusion_Argumentation_Toolkit.pdf vom 20.08.2020.

—: Inter*. Hinweise für Ärzt_innen, Psycholog_innen, Therapeut_innen \& andere medizinische Berufsgruppen 2013, https://www.transinterqueer.org/download/Publik ationen/InterSensibroschuere_2013.pdf vom 15.09.2020.

-: Redebeitrag zum Transgenialen CSD 2013. TransInterQueer-Topia 2013, https:/ /www.transinterqueer.org/download/medien/pdf/TCSD_2013_Statement.pdf vom 20.08.2020.

—: Trans*. TrIQ informiert zum Thema Transgeschlechtlichkeit. Hinweise für Ärzt_innen, Psycholog_innen, Therapeut_innen und andere Berufsgruppen aus dem Gesundheitswesen 2013, https://www.transinterqueer.org/download/Publikationen/t riq_infobroschuere_medizinpsych_berufe.pdf vom 15.09.2020.

-: Inter* und Sprache. Von >Angeboren $<$ bis $>$ Zwitter $<2015$, https://www.transinterque er.org/download/Publikationen/InterUndSprache_A_Z.pdf vom 20.08.2020.

-: Trans* in den Medien. Informationen für Journalist_innen 2015, https://www.tran sinterqueer.org/download/Publikationen/TrIQ_Journalist_innen-2.\%20Aufl.-web (2).pdf vom 20.08.2020.

-: »Danke für nix - Wie geht Christopher Street Day in solidarischen Communities?«, in: Gunda Werner Institut, https:/www.gwi-boell.de/de/2016/07/28/danke-fuer-ni $\mathrm{x}$-wie-geht-christopher-street-day-solidarischen-communities vom 24.11.2020.

-: Intersektionale Beratung von/zu Trans* und Inter*. Ein Ratgeber zu Transgeschlechtlichkeit, Intergeschlechtlichkeit und Mehrfachdiskriminierung 2016, https ://www.transinterqueer.org/wp-content/uploads/web_tis_brosch_aufl_3_161229.p df vom 20.08.2020.

-: Medizinische Eingriffe an Inter* und deren Folgen: Fakten \& Erfahrungen 2016, https://www.transinterqueer.org/download/Publikationen/eingriffe_broschuere_i nter_2016_sm.pdf vom 15.09.2020.

TransInterQueer e.V./Gladt e.V.: Pressemitteilung von TransInterQueer e.V. 2013, https: //www.transinterqueer.org/download/pm/PM_Stadtfest2013.pdf vom 20.08.2020.

Türkis Rosa Lila Villa: Abartige gegen Abschiebung, https://dievilla.at/blog/abartige-ge gen-abschiebung/ vom 14.09.2020.

—: Asyl, https://dievilla.at/asyl/ vom 20.08.2020.

-: Beratungen und Gruppen, https://dievilla.at/beratung-und-gruppen/ vom 20.08. 2020.

—: Blog, https://dievilla.at/blog/ vom 16.09.2020.

—: Coming-out, https://dievilla.at/coming-out/ vom 24.11.2020.

—: Freiräumchen, https://dievilla.at/freiraeumchen/ vom 20.08.2020. 
—: Geschichte, https://dievilla.at/geschichte/ vom 20.08.2020.

—: Lila Tipp, https://dievilla.at/lila-tipp/ vom 20.08.2020.

—: Schatzi, wir lassen uns nicht einschüchtern!, https://dievilla.at/blog/schatzi-wir-la ssen-uns-nicht-einschuchtern/ vom 20.08.2020.

—: Türkis Rosa Tipp, https://dievilla.at/traschq/ vom 20.08.2020.

—: Villa Vida, https://villavida.at/ vom 20.08.2020.

—: Wohnsatellit, https://dievilla.at/wohnsatellit/ vom 20.08.2020.

—: 20 Jahre Rosa Lila Villa 2002.

-: 15 Jahre Rosa Lila Villa 2007.

-: 30 Jahre Rosa Lila Villa 2012.

—: „Coming-out im Beruf! «, in: Lambda-Nachrichten vom Mai-Juni 2014, https://lamb danachrichten.at/ln142.pdf vom 20.08.2020, S. 14.

—: „Willkommen im Korridor! Parade 2014«, in: Lambda-Nachrichten vom Juli-August 2014, https://lambdanachrichten.at/ln143.pdf vom 20.11.2020, S. 16.

—: »Zum Mord an Hande Öncü«, in: Lambda-Nachrichten, https://lambdanachrichten .at/ln1501.pdf vom 20.08.2020, S. 18.

Whatever: Whatever, https://www.whatever.lgbt/ vom 15.09.2020.

Wild, Leo Yannick: Mit Energie und unbändiger Lust, magazin.hiv 2016, https://magaz in.hiv/2016/09/16/voller-energie-und-unbaendiger-lust/ vom 10.10.2020. 


\section{Gender \& Queer Studies}

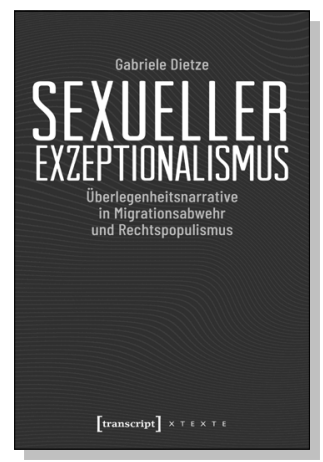

Gabriele Dietze

Sexueller Exzeptionalismus

Überlegenheitsnarrative in Migrationsabwehr

und Rechtspopulismus

2019, 222 S., kart., Dispersionsbindung, 32 SW-Abbildungen $19,99 €(D E), 978-3-8376-4708-2$

E-Book:

PDF: $17,99 €$ (DE), ISBN 978-3-8394-4708-6

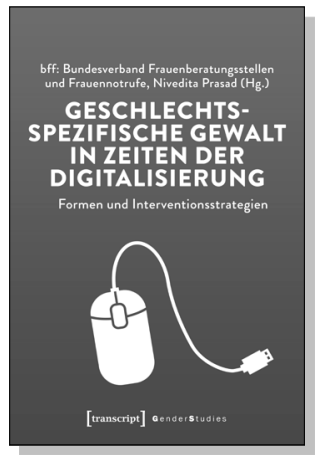

bff: Bundesverband Frauenberatungsstellen

und Frauennotrufe, Nivedita Prasad (Hg.)

Geschlechtsspezifische Gewalt in Zeiten der Digitalisierung

Formen und Interventionsstrategien

Juni 2021, 334 S., kart., Dispersionsbindung, 3 SW-Abbildungen $35,00 €(D E), 978-3-8376-5281-9$

E-Book: kostenlos erhältlich als Open-Access-Publikation

PDF: ISBN 978-3-8394-5281-3

EPUB: ISBN 978-3-7328-5281-9

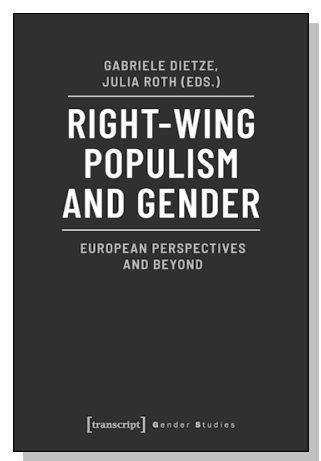

Gabriele Dietze, Julia Roth (eds.)

Right-Wing Populism and Gender

European Perspectives and Beyond

2020, 286 p., pb., ill.

$35,00 €(D E), 978-3-8376-4980-2$

E-Book:

PDF: $34,99 €$ (DE), ISBN 978-3-8394-4980-6 


\section{Gender \& Queer Studies}

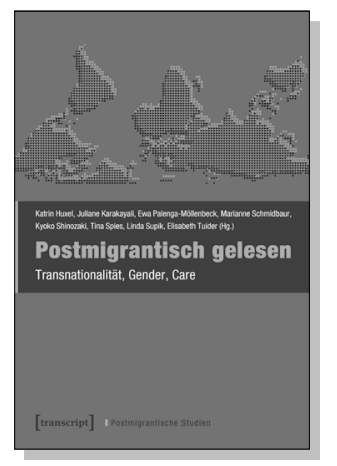

Katrin Huxel, Juliane Karakayali,

Ewa Palenga-Möllenbeck, Marianne Schmidbaur,

Kyoko Shinozaki, Tina Spies, Linda Supik, Elisabeth Tuider (Hg.)

Postmigrantisch gelesen

Transnationalität, Gender, Care

2020, 328 S., kart., Dispersionsbindung, 7 SW-Abbildungen

40,00€ (DE), 978-3-8376-4728-0

E-Book:

PDF: $39,99 €$ (DE), ISBN 978-3-8394-4728-4

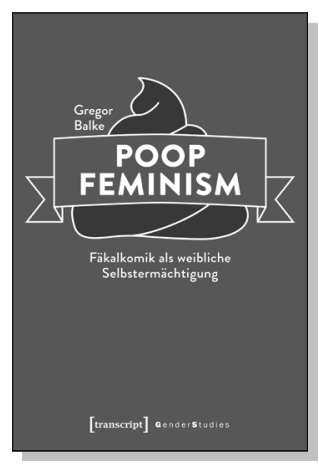

Gregor Balke

Poop Feminism -

Fäkalkomik als weibliche Selbstermächtigung

2020, 188 S., kart., Klebebindung, 30 SW-Abbildungen

$28,00 €(D E), 978-3-8376-5138-6$

E-Book:

PDF: $24,99 €$ (DE), ISBN 978-3-8394-5138-0

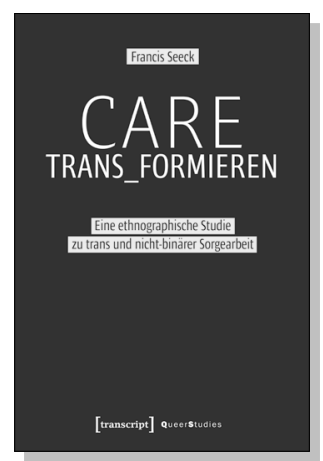

Francis Seeck

Care trans_formieren

Eine ethnographische Studie zu trans

und nicht-binärer Sorgearbeit

Juni 2021, 250 S., kart., Dispersionsbindung

25,00€ (DE), 978-3-8376-5835-4

E-Book: kostenlos erhältlich als Open-Access-Publikation PDF: ISBN 978-3-8394-5835-8 
